\title{
Biological function integrated prediction of severe radiographic progression in rheumatoid arthritis: a nested case control study
}

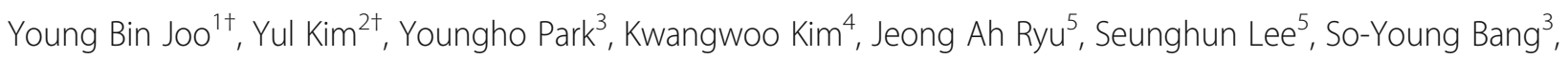
Hye-Soon Lee ${ }^{3^{*}}$, Gwan-Su Yi ${ }^{2^{*}}$ and Sang-Cheol Bae ${ }^{3^{*}}$ (D)

\begin{abstract}
Background: Radiographic progression is reported to be highly heritable in rheumatoid arthritis (RA). However, previous study using genetic loci showed an insufficient accuracy of prediction for radiographic progression. The aim of this study is to identify a biologically relevant prediction model of radiographic progression in patients with RA using a genome-wide association study (GWAS) combined with bioinformatics analysis.

Methods: We obtained genome-wide single nucleotide polymorphism (SNP) data for 374 Korean patients with RA using Illumina HumanOmni2.5Exome-8 arrays. Radiographic progression was measured using the yearly Sharp/van der Heijde modified score rate, and categorized in no or severe progression. Significant SNPs for severe radiographic progression from GWAS were mapped on the functional genes and reprioritized by post-GWAS analysis. For robust prediction of radiographic progression, tenfold cross-validation using a support vector machine (SVM) classifier was conducted. Accuracy was used for selection of optimal SNPs set in the Hanyang Bae RA cohort. The performance of our final model was compared with that of other models based on GWAS results and SPOT (one of the post-GWAS analyses) using receiver operating characteristic (ROC) curves. The reliability of our model was confirmed using GWAS data of Caucasian patients with RA.
\end{abstract}

Results: A total of 36,091 significant SNPs with a $p$ value $<0.05$ from GWAS were reprioritized using post-GWAS analysis and approximately 2700 were identified as SNPs related to RA biological features. The best average accuracy of ten groups was 0.6015 with 85 SNPs, and this increased to 0.7481 when combined with clinical information. In comparisons of the performance of the model, the 0.7872 area under the curve (AUC) in our model was superior to that obtained with GWAS (AUC 0.6586, $p$ value $8.97 \times 10^{-5}$ ) or SPOT (AUC 0.7449, $p$ value 0.0423 ). Our model strategy also showed superior prediction accuracy in Caucasian patients with RA compared with GWAS ( $p$ value 0.0049) and SPOT ( $p$ value 0.0151).

Conclusions: Using various biological functions of SNPs and repeated machine learning, our model could predict severe radiographic progression relevantly and robustly in patients with RA compared with models using only GWAS results or other post-GWAS tools.

Keywords: Rheumatoid arthritis, Radiographic progression, Bioinformatic analysis, GWAS, Post-GWAS analysis

\footnotetext{
* Correspondence: Ihsberon@hanyang.ac.kr; Ihsberon@hanyang.ac.kr; gwansuyi@kaist.ac.kr; scbae@hanyang.ac.kr

${ }^{\dagger}$ Equal contributors

${ }^{3}$ Department of Rheumatology, Hanyang University Hospital for Rheumatic

Diseases, Seoul, Republic of Korea

${ }^{2}$ Department of Bio and Brain Engineering, Korea Advanced Institute of

Science and Technology, Daejeon, Republic of Korea

Full list of author information is available at the end of the article
} 


\section{Background}

The marked success of genome-wide association studies (GWAS) has led to the discovery of numerous novel genetic loci. To date, nearly 100 susceptibility loci of rheumatoid arthritis (RA) have been identified [1]. Recently, the role of post-GWAS analysis, which prioritizes GWAS signals by incorporating diverse biological and functional evidence, has been highlighted in the identification of causal loci and for prediction of phenotypic traits [2]. Most genome-wide association loci are in noncoding regions of the genome and might not directly implicate functional variants, whereas the prioritized loci in post-GWAS analysis are biologically relevant variants and more likely to be truly associated with phenotypic traits [2].

Radiographic severity is a pivotal outcome of RA. Prediction of patients who will ultimately develop severe radiographic progression in the initial stage of the disease course is important for better outcomes and necessary for precision medicine. As radiographic severity is reported to be highly heritable (45-58\%) [3], genetic loci or genes could be helpful in the prediction of radiographic severity. However, there is currently a lack of genetic information for prediction of radiographic damage. According to a report by van Steenbergen et al., prediction accuracy of severe radiographic progression reached only $62 \%$ using a model consisting of 17 known genetic loci from several replication studies and metaanalysis and clinical factors [4].

Therefore, we sought to develop a more accurate and reliable prediction model for radiographic progression using a comprehensive approach consisting of GWAS, post-GWAS analysis, and bioinformatics. We first conducted GWAS of radiographic progression in Korean patients with early RA. Next, single nucleotide polymorphisms (SNPs) conferred by GWAS were mapped and prioritized according to their biological features through a post-GWAS approach and an optimal set of SNPs for prediction of radiographic progression was selected via tenfold cross-validation using a support vector machine (SVM). Next, a prediction model for radiographic progression was generated by the ensemble approach using genetic and clinical factors. Finally, we confirmed the usefulness of post-GWAS prioritization and our model strategy for prediction of radiographic progression in an independent cohort of Caucasian patients with RA.

\section{Methods}

\section{Patients}

All patients fulfilled the 1987 revised American College of Rheumatology criteria [5], and were recruited after providing informed consent and with ethical approval from the Institutional Review Board of Hanyang University Hospital (HYG-14-032-1).
Two cohorts were used to establish the prediction model of severe radiographic progression and their clinical characteristics are shown in Additional file 1: Table S1. First, 374 patients with early RA from the Hanyang Bae RA cohort of Hanyang University Hospital for Rheumatic Diseases [6] with two hand X-rays were included for the initial approach of post-GWAS analysis and construction of a prediction model. Next, reliability of post-GWAS prioritization for prediction of severe radiographic progression was evaluated in 399 patients with RA from the North American Rheumatoid Arthritis Consortium (NARAC) [7] with one hand X-ray per person.

\section{Radiographic outcome}

Radiographic joint damage was measured using the Sharp/Van der Heijde modified score (SHS) from hand radiographs [8]. For analysis of the Hanyang Bae RA cohort with two hand X-rays, the yearly radiographic joint damage rate $(\triangle \mathrm{SHS} /$ year) was calculated as the difference in SHS between baseline and follow-up radiographs, divided by the duration between the two X-rays. Two independent expert radiologists scored the radiographs and the interclass observer correlation coefficient was 0.89 for the total score. For analysis of the NARAC cohort with one X-ray, the estimated yearly progression rate was calculated (total SHS/disease years at time of $\mathrm{X}$-ray) as explained in a previous study [9]. Trained readers at the Leiden University Medical Center scored radiographs and the intra-observer reliability was 0.99 [10]. Patients with RA were classified into three groups of low, middle, and high tertiles based on their radiographic severity. Only the two groups of low tertile (no progression) and high tertile (severe progression) were used for analysis.

\section{Genotyping}

In the Hanyang Bae RA cohort, genotyping was conducted with Illumina HumanOmni2.5Exome-8 BeadChips at SNP Genetics Inc. (Seoul, South Korea). All subjects were successfully genotyped for $>2.5$ million markers with reliable genotyping call rates per sample $\geq 95 \%$. After the quality control, approximately 1.4 million markers with minor allele frequency (MAF) $\geq 0.5 \%$, genotyping call rate rates per each marker $\geq 95 \%$, and Hardy Weinberg equilibrium $(\mathrm{HWE})>5 \times 10^{-7}$ were used in subsequent analyses. Genetic relationship analysis performed to identify cryptic relatedness among the subjects did not find any duplicates, twins, or first-degree relatives. Principal component (PC) analysis was performed to obtain PCs and assess population stratification among the subjects. We noted that there were no genetic outliers of $>6$ standard deviations for each of the top ten PCs.

In the NARAC cohort, genotyping was conducted with Illumina BeadChips (HumanHap 550 k) [7]. As reported 
in a previous study [10], 391,733 SNPs with reliable genotyping success rate $(>98 \%)$, MAF $>0.1 \%$, and $>1 \times 10^{-5}$ were used in analyses.

\section{Genome-wide association study and genome mapping based on functional regions and eQTL data}

A comprehensive approach including GWAS, postGWAS analysis, repeated machine learning using SVM, and ensemble model was conducted to identify a prediction model for severe radiographic progression. The study workflow is presented in Fig. 1.

First, GWAS was performed in a nested case-control design, yielding genetic predictors for severe radiographic progression. Next, we mapped the statistically significant SNPs ( $p$ value $<0.05$ in GWAS analysis) with their biologically related genes based on the functional regions these SNPs map to. For this, we collected functional regions of SNPs from several public databases and obtained a total of 43,011 enhancer regions and associated genes retrieved from the FANTOM5 consortium [11]. A total of 50,900 gene regions, including both coding and intron regions and promoter regions, defined as $2 \mathrm{k}$ bases upstream from the transcription start site, were downloaded from the UCSC table browser [12]. In addition, we collected 4666 miRNA regions from miRbase [13] and their target genes from miRTarBase [14]. Moreover, we assessed cis and trans-expression quantitative trait loci (eQTL) effects by reference to four publicly available datasets [15-18]. We integrated eQTL information tested in peripheral blood mononuclear cells
(PBMCs), monocytes, CD4+ T cells, and lymphoblastoids with significance threshold defined in reference papers. When mapping the SNPs, we also considered their proxy SNPs with $\mathrm{r} 2>0.8$. Reference pair-wise linkage disequilibrium (LD) information was retrieved from HapMap genotype information of Japanese and Han Chinese populations.

\section{SNP reprioritization based on RA network}

We reprioritized the statistically significant SNPs in GWAS based on RA correlation scores of their related genes. To measure the RA correlation of the genes, we first constructed a RA gene network by propagation of prior RA information to their interaction partners (Fig. 2a). To construct the network, we used an integrated gene interaction database called HIPPIE [19], which provided 221,331 interactions between 15,615 genes. We collected prior gene-disease association (GDA) from DisGeNet [20] and disease similarity (DS) from MimMiner [21] to consider not only RA genes, but also genes for RA-related diseases. Next, for a gene $\mathrm{v}$ in the $\mathrm{Y}$ was assigned as below:

$$
\mathrm{Y}(\mathrm{v})=\operatorname{Max}\left(\mathrm{GDA}_{-}(\mathrm{v}, \mathrm{d}) \times \mathrm{DS}_{-}(\mathrm{d}, \mathrm{RA})\right),
$$

where $\mathrm{d}$ represents all disease that is associated with gene v. With assignment of prior RA information, we propagated the information using the PRINCE method [22] and calculated RA correlation scores of all genes in the network. With the RA correlation scores of genes,

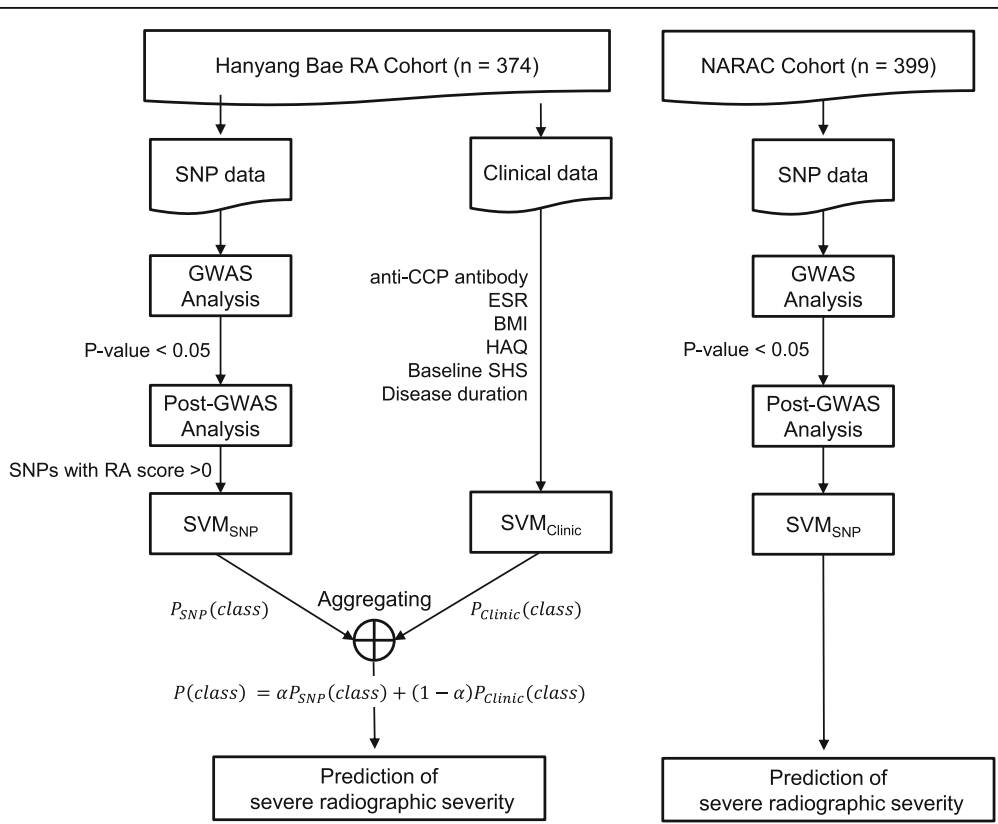

Fig. 1 Study workflow. BMI body mass index, CCP cyclic citrullinated peptide, ESR erythrocyte sedimentation rate, GWAS genome-wide association studies, HAQ health assessment questionnaire, NARAC North American Rheumatoid Arthritis Consortium, SHS Sharp/Nan der Heijde modified score, SNP single nucleotide polymorphism, SVM support vector machine 


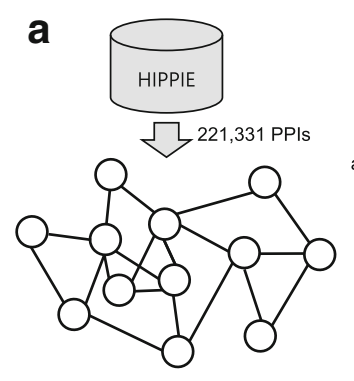

1. Construct the PPI network by using HIPPIE Database

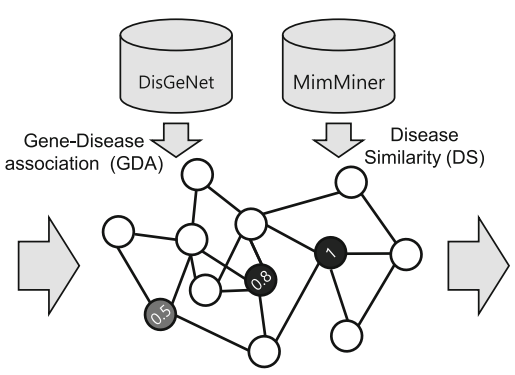

2. Assign the initial node scores based on RA-related evidences (the darker color, the higher score)

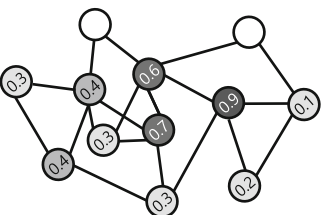

3. A scoring function is computed by using an iterative network propagation, called PRINCE algorithm

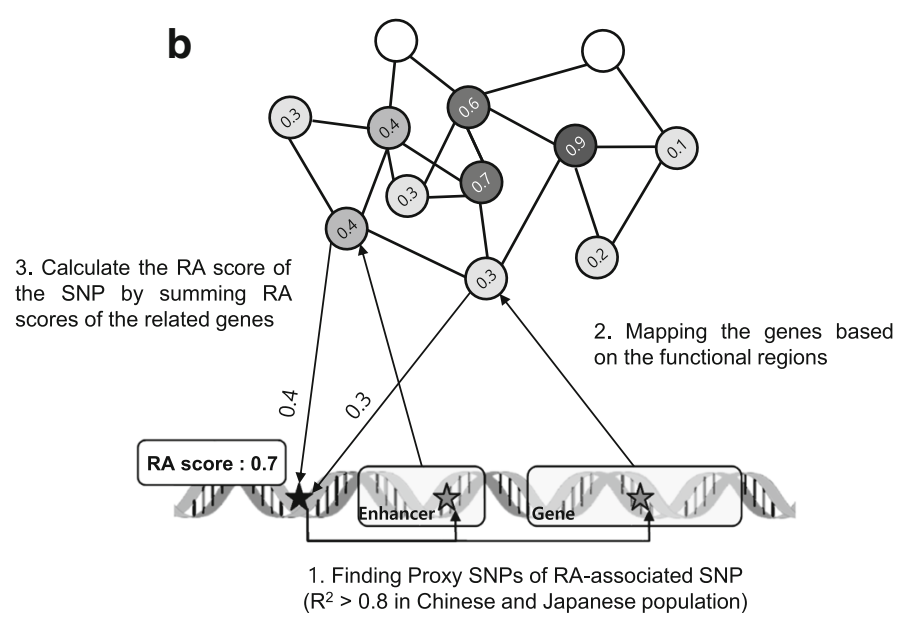

Fig. 2 Post-GWAS analysis: construction of an RA network (a), mapping statistically significant SNPs with their biological-related genes and calculation of RA correlation score of all genes in the network (b). RA rheumatoid arthritis, SNP single nucleotide polymorphism

we finally reprioritized SNPs by the sum of RA correlation scores of their related genes (Fig. 2b). We also collected SNP sets that were prioritized by $p$ value in GWAS analysis and by SPOT analysis [23] for the comparison of prediction powers.

\section{Prediction model for radiographic severity using ensemble approach}

The final prediction model for severe radiographic progression consisted of an ensemble approach that combined two classification models: one was based on the SNPs that we selected and the other was based on clinical information. Each model was constructed by using SVM, which is a supervised machine learning algorithm to classify multiclasses based on a hyperplane that differentiate the classes on the $\mathrm{n}$-dimensional space. We used six clinical predictive factors that we investigated in another study [24]: baseline SHS, disease duration, health assessment questionnaire (HAQ) index, anticyclic citrullinated peptide (CCP) antibody, body mass index (BMI), and erythrocyte sedimentation rate (ESR). The final decision for severe radiographic progression was calculated as the weight sum of probabilities in each model.

\section{Post-GWAS prioritization in an independent cohort of Caucasian patients with RA}

To confirm the reliability of post-GWAS prioritization and SVM-based prediction of severe radiographic progression, we conducted GWAS, post-GWAS analysis, and machine learning using SVM in consecutive order. As there was limited clinical information in the NARAC cohort, only SNPs were used for prediction of severe radiographic progression.

\section{Statistical analysis}

The multivariate logistic regression model was used to investigate the association between SNPs and radiographic severity (no progression vs. severe progression) in GWAS, adjusted for anti-CCP antibody positivity, ESR, BMI, HAQ score, baseline SHS, disease duration, and the top ten principal components using PLINK v1.07. 
Accuracy is a measure of the proportion of samples that are correctly predicted among all the test samples, and it is easy to intuitively understand the model performance at a glance. Thus, accuracy was used for selection of optimal SNPs set in the Hanyang Bae RA cohort and NARAC cohort, according to the standard method as follows:

$$
\text { Accuracy }=\frac{\sum \text { True positive }+\sum \text { True negative }}{\sum \text { True population }}
$$

Classification accuracy is typically not enough information to evaluate the performance of the model. To evaluate the robustness of a model, more performance measures are needed. The area under the curve (AUC) of the receiver operating characteristic (ROC) curve measures the performance of the markers with the total sum of performance at all thresholds. Based on the diversity of populations and the characteristics of SNP markers that should be evaluated with a limited number of samples, it would be more reliable to compare all the performance that the set could have, rather than looking for the best accuracy it could have, with expectations for performance for various unknown samples. In this reason, we further analyzed the performance of the model in the Hanyang Bae RA cohort using AUC according to the standard method as follows:

$$
\mathrm{AUC}=\frac{\sum \operatorname{Rank}(\text { pos })-\# \text { pos } \times(\# \text { pos }+1) / 2}{\# \text { pos }+\# \text { neg }}
$$

Where $\Sigma \operatorname{Rank}($ pos) means the sum of the ranks of all positively classified examples, \#pos means the number of positive examples in the dataset, and \#neg means the number of negative examples in the dataset.

\section{Results}

Findings of GWAS for radiographic progression in Korean RA patients

After quality control, a total of 1,343,748 SNPs were available for comparison in 118 patients with no progression [age $49.5 \pm 11.8$ (mean \pm standard deviation), female $=83.9 \%$ ] and 120 patients with severe progression [age $47.7 \pm 12.6$, female $=85.0 \%$ ] (Additional file 1: Table S1). In the single association analysis, none of the SNPs reached the significance threshold after Bonferroni correction. The SNPs with $p<1.0 \times 10^{-3}$ and their related genes are listed in Additional file 1: Table S2.

\section{Optimal SNP set selection using post-GWAS scoring}

To determine the optimal number of SNPs for the prediction model, we tested the accuracy of the prediction model by adding 5 SNPs from the top ten scored SNPs. For this, we performed tenfold cross-validation by grouping the patients into ten groups. Of the ten groups, nine groups were used as the training set in GWAS and post-GWAS analysis for selection of the SNPs and construction of a SVM model using radial basis function Kernel. The remaining group was used as a test set and we calculated the average accuracies of ten test sets. Our results showed that the best accuracy was 0.6015 when the top 85 SNPs were used (Fig. 3a). Therefore, we defined the optimal number of SNPs as 85. Our method showed superior accuracy compared with SNPs selected based on $\mathrm{p}$-value of GWAS and by SPOT analysis ( $p$ value $1.06 \times 10^{-06}$ and $6.25 \times 10^{-03}$, respectively). The list of $85 \mathrm{SNPs}$ and their related genes are described in Additional file 1: Table S3.

Interestingly, SNPs that had low $p$ values in GWAS analysis showed the lowest accuracy. To investigate further, we compared the overlapping ratio of the top 85 SNPs selected by different methods between ten training sets (Fig. 3b). The results showed that the overlap ratio between SNPs selected by low $p$ value was only 0.2403 , whereas the overlap ratio of our method was more than two times higher (0.5627). It seems that the SNPs selected in each training set by tenfold cross-validation based on GWAS $p$ value are likely to be biased in each training sample itself and could cause lower overlap ratio between groups. On other hand, post-GWAS analysis which integrated the biological meaning to the analysis is less likely to select the SNPs that were biased in the sample group, unlike the GWAS which depends only on the simple $p$ value.

\section{Comparison of the prediction accuracy among different models}

The final ensemble model was composed of two different SVM models: one is based on 85 SNPs selected by post-GWAS analysis and the other is based on information on six clinical factors. We calculated a weighted sum of probabilities of these two models to predict severe radiographic progression. The best average accuracy of our model was 0.7481 with 0.27 of weight to SVM model using SNPs (Additional file 2: Figure S1). In the process of optimizing the weight, all tenfold cross-validation tests were performed on the test set, to avoid overfitting as much as possible. We compared the ROC curve of our ensemble model with other ensemble models that used 85 SNPs selected by GWAS $p$ value or by SPOT analysis as well as clinical information (Fig. 4). The AUC of our model was 0.7872 (sensitivity 0.7644, specificity 0.7318 , and positive predictive value 0.7445 , Additional file 1 : Table S4), which was significantly better than that of the ensemble model with GWAS (8.97X10E-5) and SPOT (0.0423). 


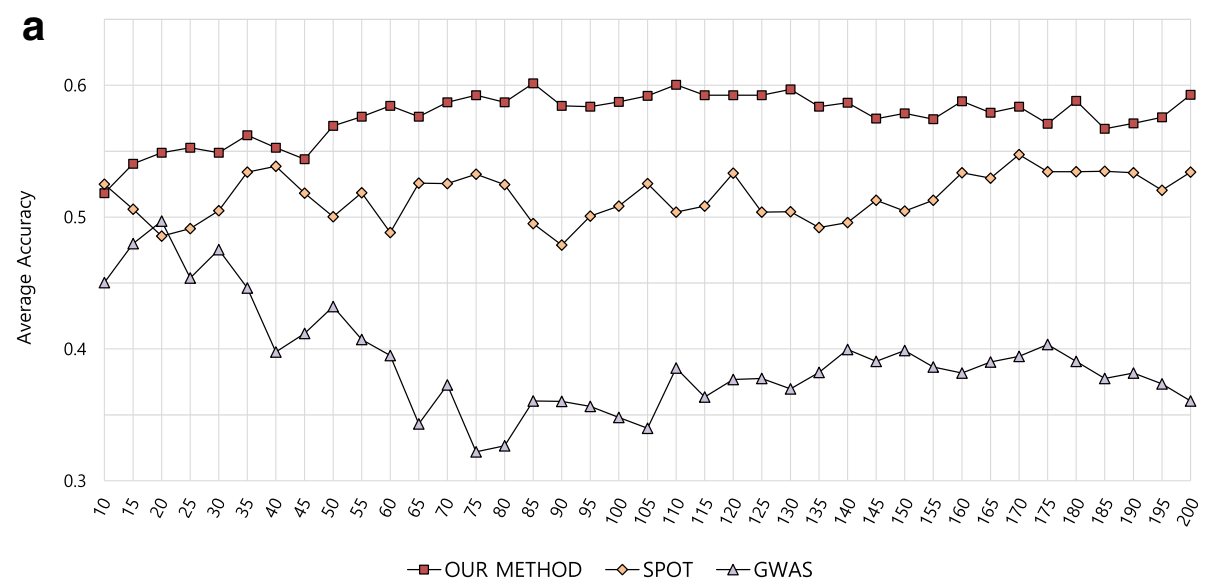

b

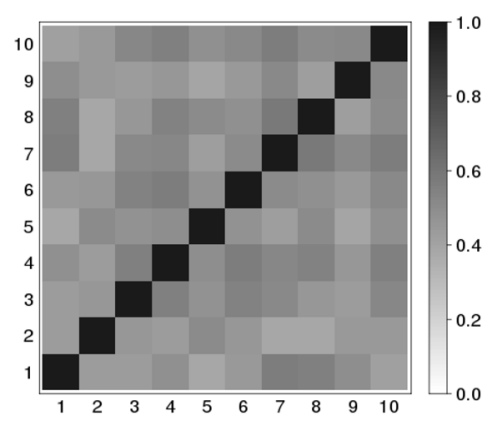

OUR METHOD (post-GWAS)

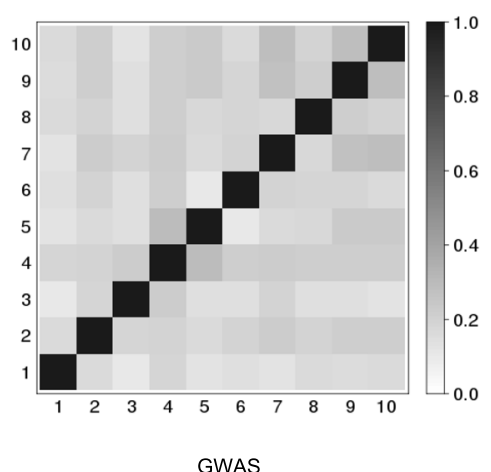

GWAS

Fig. 3 Prediction accuracy of radiographic progression using SNPs obtained via post-GWAS, GWAS, and SPOT analysis: optimal number of SNPS for the prediction model (a), and overlapping ratio between 85 SNPs selected by post-GWAS and GWAS analysis (b). GWAS genome-wide association studies

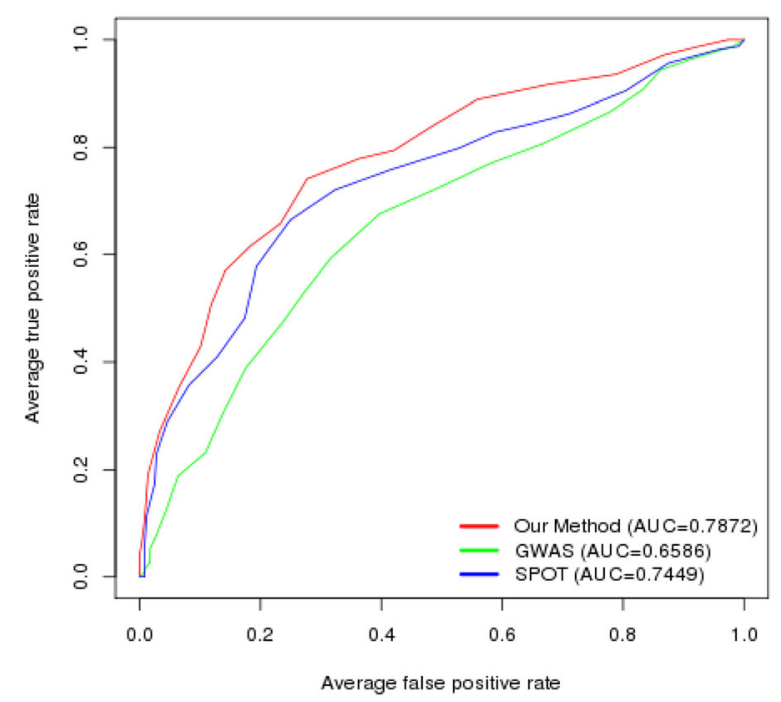

Fig. 4 Comparison of prediction accuracy of our ensemble model with other methods in Korean patients with RA. AUC area under the curve, GWAS genome-wide association studies
Reliability of post-GWAS prioritization in the independent cohort

By applying the same methods of post-GWAS prioritization and tenfold cross-validation using SVM to the NARAC cohort (68 patients with no progression and 86 patients with severe progression), we were able to confirm that the SNPs selected by post-GWAS analysis were more accurate than those selected by statistical significance in GWAS for prediction of severe radiographic progression. In the NARAC cohort the average accuracy was 0.6143 with SNPs selected by post-GWAS analysis, which was superior to that using SNPs selected by statistical significance in GWAS (average accuracy 0.3875 ) or by SPOT analysis (average accuracy 0.4563) (Fig. 5).

After quality control, a total of 1,343,748 SNPs were available for comparison in 118 patients with no progression [age $49.5 \pm 11.8$ (mean \pm standard deviation), female $=$ $83.9 \%$ ] and 120 patients with severe progression [age $47.7 \pm$ 12.6, female $=85.0 \%$ (Additional file 1: Table S1).

\section{Discussion}

As hypothesized in this study, our new model allowed us to conduct more relevant and robust prediction of 


\section{$P$ value 0.0049}

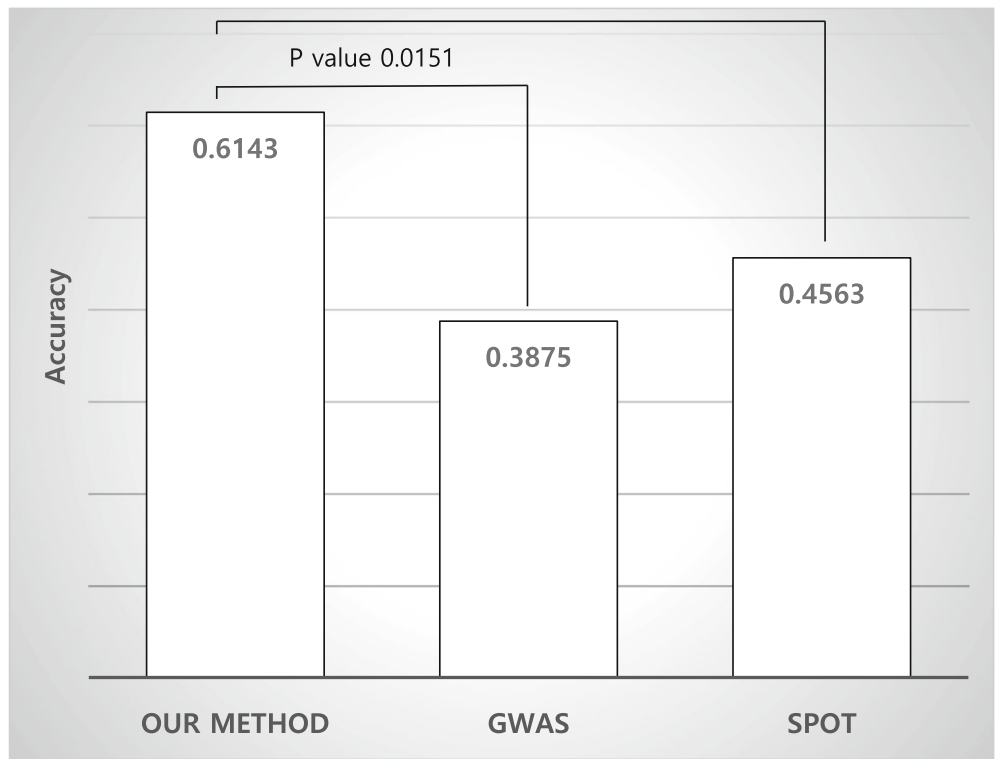

Fig. 5 Comparison of prediction accuracy of our model with other methods in an independent Caucasian cohort. GWAS genome-wide association studies

radiographic severity in RA. In short, using post-GWAS analysis we identified biologically relevant SNPs related to RA progression in patients with early RA. Our final model composed of SNPs combined with clinical factors could satisfactorily discriminate severe progression from the absence of progression, showing an average AUC of 0.78 in tenfold cross-validation. This result was superior to those obtained using data from GWAS (AUC $=0.59$ ) or SPOT $(A U C=0.67)$, one of the methods of postGWAS analysis. The superior effectiveness of our prediction model was also successfully reproduced in an independent cohort.

We initially thought that biological function-enriched prediction of radiographic severity would overcome the overfitting effect although the prediction accuracy would be similar to that using only GWAS results. Interestingly, however, the prediction accuracy was also improved compared with that using data of GWAS. Selection of biologically relevant variants based on postGWAS analysis, in addition to $p$ value in GWAS, and use of a machine learning algorithm such as SVM enabled more accurate and robust prediction of radiographic severity despite the limited sample size.

Regarding post-GWAS analysis, there have been examples of effective integration of biological database information of SNPs with GWAS results to identify causal SNPs in colorectal cancer [25] and chronic lymphocytic lymphoma [26]. We used information on various functional regions associated with SNPs and related genes such as enhancer region, mRNA, promoter region, miRNA region, and posttranscriptional modification
(PTM), in addition to expression of quantitative traits, and gave a higher priority to SNPs with greater involvement with these genes. Thus, SNPs with higher biological relevance obtained a higher reprioritization score and might be used in prediction of radiographic severity.

The SVM algorithm also contributed to increased prediction accuracy. It is one of the popular supervised learning techniques in classification. In the SVM algorithm, each patient is represented in $\mathrm{n}$-dimensional space where $\mathrm{n}$ is the number of SNPs. After that, it finds a hyperplane that can separate patients' classes with maximum margin. We also used Kernel trick that mapped original dimensional space into a much higherdimensional space. It can help to do a nonlinear classification more efficiently. This learning machine technique could discover new patterns for input features via investigation of complex relationships among SNPs, and thus increase the explanation power for prediction of radiographic severity in RA [27]. Many examples of outcome prediction with high predictive accuracy using SVM algorithms have been reported, such as in breast cancer [28], nasopharyngeal carcinoma [29], and severe radiationinduced pneumonitis in lung cancer [30-32]. Similarly, we could predict severe radiographic progression with high predictive accuracy via a SVM-based ensemble model that integrated multidimensional SNP data and clinical factors.

It is interesting that our model was superior to SPOT, which is also a method of SNP prioritization [23]. However, there were some differences between our SNP prioritization method and SPOT. Information on functional properties used in annotation was not the same; 
our method used more varied biological information related to SNPs and genes including transcription factor binding sites, micro RNA regions, PTM, and eQTL. An eQTL study was able to explain the functional basis of up to $50 \%$ of SNPs related to immune-mediated disease [33] and therefore might be very useful in predicting the outcome of RA. Another important difference is the characteristics of the network used for scoring. In contrast to SPOT, we used a disease-specific gene database during construction of the network based on the concept that a RA susceptibility gene is also associated with phenotype. This is the first RA-specific network constructed based on network propagation and might give more accurate and stable relationship information to reprioritize the SNPs conferred by GWAS.

This study has some limitations. First, the sample size used in the analysis was small, which could lead to lower predictive accuracy of GWAS. However, as we applied the results of GWAS to post-GWAS analysis and tenfold cross-validation we could achieve higher predictive accuracy of radiographic progression and robustness of top SNPs in each of the ten groups. This meant that post-GWAS could take advantage of the small sample size of subjects in contrast to GWAS, which needs numerous samples to identify disease-specific loci. Second, we did not use the 85 SNPs selected in the Korean cohort in the analysis of the Caucasian cohort. Rather, we reproduced all courses of analysis from GWAS to using the SVM classifier in the Caucasian cohort to show the advantage of a post-GWAS approach over GWAS as a method of prediction. When we validated the final SNPs from the Hanyang Bae RA cohort in the NARAC cohort, the performance of the model using the final SNPs was unsatisfactory. Among the 85 SNPs, 72 SNPs were identified in the NARAC cohort and the accuracy (standard deviation) of the model was $0.5062(0.1239)$ and the AUC was 0.4739 (Additional file 2: Figure S2). It seems that the same SNPs are not useful across ethnic groups for many reasons, such as ethnicity-specific SNPs or different allele frequency, or linkage disequilibrium.

\section{Conclusions}

We demonstrated that biologically relevant SNPs could provide more accurate and robust prediction of severe radiographic progression in Korean and Caucasian cohorts. Biologically relevant prediction of radiographic progression was possible through a bioinformatics approach including post-GWAS, which was conducted with functional annotation of the genome gathered from GWA studies, a RA network with propagation, and machine learning algorithm. This approach worked better than the GWAS approach alone. SNPs and genes selected in this approach could be targets for further functional studies and might be a basis of individual precision medicine.

\section{Additional files}

Additional file 1: Table S1. Characteristics of study populations. Table S2. GWAS results for severe radiographic progression $(p<1.0 \times 10-3)$. Table S3. List of the top 85 SNPs and their related genes selected by a post-GWAS approach. Table S4 Sensitivity, specificity, and positive predictive value of the final models. (DOCX $77 \mathrm{~kb}$ )

Additional file 2: Figure S1. The weighted sum of probabilities in each model. Figure S2. Receiver operating characteristic (ROC) curve for the result of replication of the final SNPs in the NARAC cohort. (PPTX $207 \mathrm{~kb}$ )

\section{Abbreviations \\ AUC: Area under the curve; BMI: Body mass index; CCP: Cyclic citrullinated peptide; eQTL: Expression quantitative trait loci; ESR: Erythrocyte sedimentation rate; GWAS: Genome-wide association studies; HAQ: health assessment questionnaire; HWE: Hardy Weinberg equilibrium; MAF: Minor allele frequency; NARAC: North American Rheumatoid Arthritis Consortium; PC: Principal component; PTM: posttranscriptional modification; RA: Rheumatoid arthritis; ROC: Receiver operating characteristic; SHS: Sharp/Van der Heijde modified score; SNP: Single nucleotide polymorphisms; SVM: Support vector machine}

\section{Acknowledgements}

The authors would like to thank Dr. Gregersen PK for kindly providing the $X$-rays and clinical data from the North American Rheumatoid Arthritis Consortium (NARAC).

\section{Funding}

This work was supported by grants from the Korea Healthcare Technology R\&D Project of the Ministry for Health \& Welfare (HI13C2124) and the Bio-Synergy Research Project (NRF-2012M3A9C4048759) of the Ministry of Science, ICT and Future Planning through the National Research Foundation.

\section{Authors' contributions}

YBJ, YK, G-SY, H-SL, and S-CB designed the study. JAR and SL scored all radiographs, $Y K$ and $Y P$ analyzed the data. YBJ, YK, KK, and S-YB interpreted the data. YBJ, YK, and S-CB wrote the manuscript. All authors reviewed and approved the manuscript.

\section{Ethics approval and consent to participate}

This study was approved by the Institutional Review Board of Hanyang University Hospital (HYG-14-032-1) and consents were obtained from all patients who participated in this study.

\section{Competing interests}

The authors declare that they have no competing interests.

\section{Publisher's Note}

Springer Nature remains neutral with regard to jurisdictional claims in published maps and institutional affiliations.

\section{Author details \\ ${ }^{1}$ Department of Rheumatology, St. Vincent's Hospital, The Catholic University of Korea, Suwon, Republic of Korea. ${ }^{2}$ Department of Bio and Brain Engineering, Korea Advanced Institute of Science and Technology, Daejeon, Republic of Korea. ${ }^{3}$ Department of Rheumatology, Hanyang University Hospital for Rheumatic Diseases, Seoul, Republic of Korea. ${ }^{4}$ Department of Biology, Kyung Hee University, Seoul, Republic of Korea. ${ }^{5}$ Department of Radiology, Hanyang University Hospital, Seoul, Republic of Korea.}

Received: 15 June 2017 Accepted: 31 August 2017

Published online: 25 October 2017

\footnotetext{
References

1. Kim K, Bang SY, Lee HS, Bae SC. Update on the genetic architecture of rheumatoid arthritis. Nat Rev Rheumatol. 2017;13:13-24.

2. Hou L, Zhao H. A review of post-GWAS prioritization approaches. Front Genet. 2013;4:280.
} 
3. Knevel R, Grondal G, Huizinga TW, Visser AW, Jonsson H, Vikingsson A, Geirsson AJ, Steinsson K, van der Helm-van Mil AH. Genetic predisposition of the severity of joint destruction in rheumatoid arthritis: a population-based study. Ann Rheum Dis. 2012:71:707-9.

4. van Steenbergen HW, Tsonaka R, Huizinga TW, le Cessie S, van der Helm-van Mil AH. Predicting the severity of joint damage in rheumatoid arthritis; the contribution of genetic factors. Ann Rheum Dis. 2015:74:876-82

5. Arnett FC, Edworthy SM, Bloch DA, McShane DJ, Fries JF, Cooper NS, Healey LA, Kaplan SR, Liang MH, Luthra HS, et al. The American Rheumatism Association 1987 revised criteria for the classification of rheumatoid arthritis. Arthritis Rheum. 1988;31:315-24.

6. Kim YJ, Choi CB, Sung YK, Lee HS, Bae SC. Characteristics of Korean patients with RA: a single center cohort study. J Korean Rheum Assoc. 2009;16:204-12.

7. Plenge RM, Seielstad M, Padyukov L, Lee AT, Remmers EF, Ding B, Liew A, Khalili $\mathrm{H}$, Chandrasekaran A, Davies LR, et al. TRAF1-C5 as a risk locus for rheumatoid arthritis-a genomewide study. N Engl J Med. 2007;357:1199-209.

8. van der Heijde D. How to read radiographs according to the Sharp/van der Heijde method. J Rheumatol. 2000;27:261-3.

9. Strand V, Landewe $R$, van der Heijde D. Using estimated yearly progression rates to compare radiographic data across recent randomised controlled trials in rheumatoid arthritis. Ann Rheum Dis. 2002;61 Suppl 2:ii64-66.

10. Knevel R, Klein K, Somers K, Ospelt C, Houwing-Duistermaat JJ, van Nies JA, de Rooy DP, de Bock L, Kurreeman FA, Schonkeren J, et al. Identification of a genetic variant for joint damage progression in autoantibody-positive rheumatoid arthritis. Ann Rheum Dis. 2014;73:2038-46.

11. Forrest AR, Kawaji H, Rehli M, Baillie JK, de Hoon MJ, Haberle V, Lassmann T, Kulakovskiy IV, Lizio M, Itoh M, et al. A promoter-level mammalian expression atlas. Nature. 2014;507:462-70.

12. Karolchik D, Baertsch R, Diekhans M, Furey TS, Hinrichs A, Lu YT, Roskin KM, Schwartz M, Sugnet CW, Thomas DJ, et al. The UCSC Genome Browser Database. Nucleic Acids Res. 2003:31:51-4.

13. Griffiths-Jones S, Grocock RJ, van Dongen S, Bateman A, Enright AJ. miRBase: microRNA sequences, targets and gene nomenclature. Nucleic Acids Res. 2006;34:D140-4.

14. Hsu SD, Lin FM, Wu WY, Liang C, Huang WC, Chan WL, Tsai WT, Chen GZ, Lee CJ, Chiu CM, et al. miRTarBase: a database curates experimentally validated microRNA-target interactions. Nucleic Acids Res. 2011:39:D163-169.

15. Xia K, Shabalin AA, Huang S, Madar V, Zhou YH, Wang W, Zou F, Sun W, Sullivan PF, Wright FA. seeQTL: a searchable database for human eQTLs. Bioinformatics. 2012;28:451-2.

16. GTEx Consortium. The Genotype-Tissue Expression (GTEx) project. Nat Genet. 2013:45:580-5.

17. Westra HJ, Arends D, Esko T, Peters MJ, Schurmann C, Schramm K, Kettunen J, Yaghootkar H, Fairfax BP, Andiappan AK, et al. Cell specific eQTL analysis without sorting cells. PLoS Genet. 2015;11:e1005223.

18. De Jager PL, Hacohen N, Mathis D, Regev A, Stranger BE, Benoist C. ImmVar project: Insights and design considerations for future studies of "healthy" immune variation. Semin Immunol. 2015:27:51-7.

19. Schaefer $M H$, Fontaine JF, Vinayagam A, Porras P, Wanker EE, Andrade-Navarro MA. HIPPIE: Integrating protein interaction networks with experiment based quality scores. PLoS One. 2012;7:e31826.

20. Pinero J, Queralt-Rosinach N, Bravo A, Deu-Pons J, Bauer-Mehren A, Baron M, Sanz F, Furlong LI. DisGeNET: a discovery platform for the dynamical exploration of human diseases and their genes. Database (Oxford). 2015;2015:bav028.

21. van Driel MA, Bruggeman J, Vriend G, Brunner HG, Leunissen JA. A text-mining analysis of the human phenome. Eur J Hum Genet. 2006;14:535-42.

22. Vanunu O, Magger O, Ruppin E, Shlomi T, Sharan R. Associating genes and protein complexes with disease via network propagation. PLoS Comput Biol. 2010;6:e1000641.

23. Saccone SF, Bolze R, Thomas P, Quan J, Mehta G, Deelman E, Tischfield JA, Rice JP. SPOT: a web-based tool for using biological databases to prioritize SNPs after a genome-wide association study. Nucleic Acids Res. 2010;38:W201-209.

24. Joo YB, Bang SY, Ryu JA, Lee S, Lee HS, Bae SC. Predictors of severe radiographic progression in patients with early rheumatoid arthritis: Prospective observational cohort study. Int J Rheum Dis. 2017. doi:10.1111/ 1756-185X.13054. [Epub ahead of Print]
25. Wang HM, Chang TH, Lin FM, Chao TH, Huang WC, Liang C, Chu CF, Chiu CM, Wu WY, Chen MC, et al. A new method for post Genome-Wide Association Study (GWAS) analysis of colorectal cancer in Taiwan. Gene. 2013:518:107-13.

26. Sille FC, Thomas R, Smith MT, Conde L, Skibola CF. Post-GWAS functional characterization of susceptibility variants for chronic lymphocytic leukemia. PLoS One. 2012;7(e29632)

27. Klement RJ, Allgauer M, Appold S, Dieckmann K, Ernst I, Ganswindt U, Holy R, Nestle U, Nevinny-Stickel M, Semrau S, et al. Support vector machine-based prediction of local tumor control after stereotactic body radiation therapy for early-stage non-small cell lung cancer. Int J Radiat Oncol Biol Phys. 2014;88:732-8

28. Nimeus-Malmstrom E, Krogh M, Malmstrom P, Strand C, Fredriksson I, Karlsson P, Nordenskjold B, Stal O, Ostberg G, Peterson C, et al. Gene expression profiling in primary breast cancer distinguishes patients developing local recurrence after breast-conservation surgery, with or without postoperative radiotherapy. Breast Cancer Res. 2008;10:R34

29. Wan XB, Zhao Y, Fan XJ, Cai HM, Zhang Y, Chen MY, Xu J, Wu XY, Li HB, Zeng $Y X$, et al. Molecular prognostic prediction for locally advanced nasopharyngeal carcinoma by support vector machine integrated approach. PLoS One. 2012;7(e31989).

30. Naqa IE, Deasy JO, Mu Y, Huang E, Hope AJ, Lindsay PE, Apte A, Alaly J, Bradley JD. Datamining approaches for modeling tumor control probability. Acta Oncol. 2010:49:1363-73.

31. Chen S, Zhou S, Yin FF, Marks LB, Das SK. Investigation of the support vector machine algorithm to predict lung radiation-induced pneumonitis. Med Phys. 2007;34:3808-14.

32. Das SK, Chen S, Deasy JO, Zhou S, Yin FF, Marks LB. Combining multiple models to generate consensus: application to radiation-induced pneumonitis prediction. Med Phys. 2008;35:5098-109.

33. Kumar V, Wijmenga C, Xavier RJ. Genetics of immune-mediated disorders: from genome-wide association to molecular mechanism. Curr Opin Immunol. 2014:31:51-7.

\section{Submit your next manuscript to BioMed Central and we will help you at every step:}

- We accept pre-submission inquiries

- Our selector tool helps you to find the most relevant journal

- We provide round the clock customer support

- Convenient online submission

- Thorough peer review

- Inclusion in PubMed and all major indexing services

- Maximum visibility for your research

Submit your manuscript at www.biomedcentral.com/submit 